\title{
Implementación de la estrategia "Atención Limpia es Atención Segura" en un hospital de tercer nivel en Medellín, Colombia
}

\author{
Andrea V. Restrepo, Mónica P. Valderrama, Ana L. Correa, Luz M. Mazo, Nancy E. González y Fabián Jaimes
}

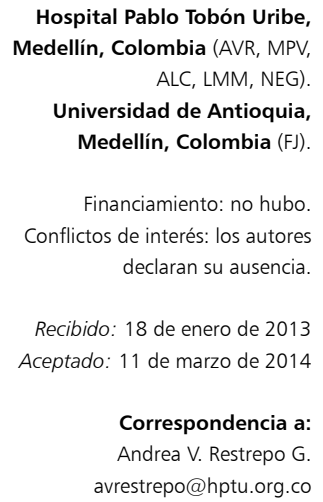

\section{Implementation of the strategy "Clean Care is Safer Care" in a third level hospital in Medellín, Colombia}

Introduction: Hand hygiene is the most cost-effective and simple measure of preventing healthcare associated infections (HAI). The approach to improve low compliance must be through multimodal interventions such as the "Clean Care is Safer Care" strategy (WHO). Aim: To estimate the efficacy of a multimodal strategy in improving hand hygiene in five wards of a tertiary care hospital in Medellín, Colombia (2008-2010). Methods: Quasi-experimental before-after study. Results: Hand hygiene compliance significantly increased after the intervention ( 82 to $89 \%, \mathrm{p}=0.007)$. The knowledge score increased from a median of $26(\mathrm{IQR}=22-28)$ to $30(\mathrm{IQR}=26-32, \mathrm{p}=0.001)$. Alcohol-based hand rub consumption increased significantly from 10.5 liters to 58.1 liters per 1000 patient-days [incidence ratio $(\mathrm{IR})=2.39,95 \% \mathrm{CI}=1.99 ; 2.88$ ]. Monthly HAI rates showed no significant variations during the same period [IR $=0.90,95 \% \mathrm{CI}=0.71 ; 1.13]$. Discussion: This and other recent studies demonstrate that implementing a multimodal strategy for hand hygiene significantly increases compliance with this measure, irrespective of type of health worker or hospital department. Conclusions: Implementing a multimodal strategy we achieved significant increases in hand hygiene compliance but mild or no significant variations in monthly HAI rates.

Key words: Clean care is safer care, hand hygiene, alcohol-based hand rub, health-care associated infections, multimodal strategy.

Palabras clave: Atención limpia es atención segura, higiene de manos, desinfectante alcohólico para las manos, infecciones asociadas a la atención en salud, estrategia multimodal.

\section{Introducción}

L as infecciones asociadas a la atención en salud (IAAS) constituyen el efecto adverso más frecuente durante el cuidado de los pacientes. La prevalencia de estas infecciones varía significativamente según cada centro hospitalario; se calcula una prevalencia general de $10,1 \%{ }^{1}$. En países de medianos y bajos recursos la carga de la enfermedad por infecciones es más alta, razón por que es prioritario establecer políticas nacionales para la prevención de dichos eventos ${ }^{1}$.

La higiene de manos es la medida más costo-efectiva y sencilla para prevenir las IAAS ${ }^{2,3}$. Implica una actitud de solidaridad y responsabilidad con el paciente y un hábito que requiere práctica. A pesar de ser una medida fácil de realizar, el cumplimiento por parte de los trabajadores de la salud es bajo y depende de varios factores como su nivel de conocimiento, las campañas de promoción, la carga laboral, la disponibilidad de productos y los estímulos, entre otros ${ }^{4,5}$. Debido a que este problema es multifactorial, se requiere una estrategia multimodal para abordarlo $0^{6,7}$. La estrategia multimodal "Atención Limpia es Atención Segura", es la primera iniciativa mundial en pro de la seguridad del paciente, está liderada por la Organi- zación Mundial de la Salud (OMS) y en Colombia por el Ministerio de Salud ${ }^{7,8}$. Su principal objetivo es aumentar el cumplimiento con la higiene de manos. El Hospital Pablo Tobón Uribe (HPTU) asumió el compromiso de desarrollar la estrategia desde noviembre de 2008. El objetivo de este estudio es describir la implementación y los resultados preliminares de la estrategia "Atención Limpia es Atención Segura", desarrollada en cinco servicios del HPTU durante un período de 20 meses.

\section{Métodos}

\section{Sitio del estudio}

El HPTU (Medellín, Colombia) es un hospital universitario de tercer nivel de complejidad que cuenta con 380 camas. Dispone de todas las especialidades médico-quirúrgicas, excepto obstetricia; es un centro de trasplantes de precursores hematopoyéticos, hígado, riñón y multi-visceral. Para la intervención se eligieron cinco servicios hospitalarios: unidades de cuidado crítico (pediátrico y adulto), unidades de cuidado intermedio (pediátrico y adulto) y la unidad de aislamiento para pacientes con infección por microorganismos multi-resistentes $\left(8^{\circ}\right.$ piso sur), las cuales contienen un total de 80 camas. 


\section{Diseño del estudio}

Estudio cuasi-experimental de antes y después de la implementación de la estrategia multimodal "atención limpia es atención segura". Durante el período comprendido entre noviembre de 2008 y julio de 2010 se implementó la estrategia en los cinco servicios mencionados. Esta estrategia se basa en cinco elementos:

- Cambio de sistema, que se refiere al uso preferencial de desinfectante alcohólico para la higiene de manos.

- Educación y entrenamiento a los trabajadores de la salud.

- Medición del cumplimiento mediante observaciones advertidas y retroalimentación inmediata.

- Afiches y recordatorios en el sitio de trabajo.

- Creación del clima de seguridad institucional.

La intervención se desarrolló según la "Guía para la Aplicación" de la $\mathrm{OMS}^{9}$ y consta de cinco pasos consecutivos:

- Preparación del sitio: se conformó un grupo coordinador y se garantizó el compromiso de las directivas y jefes de los servicios seleccionados. Se decidió mantener el desinfectante alcohólico para las manos marca Purell ${ }^{\mathrm{TM}}$ por la alta aceptación que tenía en el personal.

- Evaluación inicial: se evaluaron la percepción y los conocimientos de los directivos y los trabajadores del hospital sobre la importancia e impacto de la higiene de manos en las IAAS. Se entrenaron 30 observadores mediante vídeos que reproducen situaciones reales de la atención hospitalaria. A cada observador se le realizó una prueba de concordancia, con base en las respuestas correctas a las acciones reproducidas en los vídeos, y se consideró aceptable un índice de concordancia kappa de al menos 0,6 entre los observadores y el estándar. Aquellos que obtuvieron valores inferiores (9 participantes) fueron re-entrenados y evaluados nuevamente una semana después. Luego del entrenamiento se realizaron las primeras observaciones para hacer el cálculo del cumplimiento inicial ("antes"), las cuales estuvieron acompañadas de una retroalimentación inmediata. Se midió el consumo de desinfectante alcohólico para las manos y de jabones microbicidas durante toda la intervención.

- Aplicación de la estrategia: durante este paso se realizó el lanzamiento de la estrategia mediante actividades lúdico-pedagógicas y el entrenamiento en el modelo "Mis cinco momentos para la higiene de manos" a todos los trabajadores de las unidades seleccionadas. El entrenamiento incluyó sensibilización sobre la importancia de la higiene de manos, utilización de ayudas audiovisuales y entrega de plegables sobre cómo, cuándo y porqué realizar higiene de manos ${ }^{10}$. Se instalaron los afiches de "Mis cinco momentos" en todas las habitaciones y los de cómo realizar fricción $\mathrm{y}$ lavado en todos los dispensadores y lavamanos. Se realizó un concurso para estimular el cumplimiento

- Evaluación, seguimiento y retroalimentación: se realizaron las observaciones y las encuestas sobre conocimientos y percepciones de la estrategia, correspondientes al período "después" de la intervención y se brindó retroalimentación a los trabajadores según los datos obtenidos.

- Desarrollo de un plan de acción continuo: se diseñó el plan para continuar la implementación de la estrategia en el resto del hospital.

\section{Análisis estadístico}

Las variables se presentan como medianas (Me) o medias (M) con rango intercuartílico (RIC) o desviación estándar (DS), respectivamente, en el caso de datos continuos y como proporciones para el caso de variables categóricas. El puntaje de conocimientos ${ }^{10}$ acerca de la higiene de manos para cada participante se calculó con base en el número total de respuestas correctas, otorgando un punto por respuesta para un máximo posible de 46 puntos. Dado que las respuestas fueron anónimas y no necesariamente se realizaban en las mismas personas antes y después de la estrategia, no era posible identificar los individuos para hacer una prueba pareada de Wilcoxon. Por lo tanto, la comparación entre la mediana de los resultados de la prueba de conocimientos antes y después de la implementación de la estrategia se hizo por medio de la prueba de Mann-Whitney.

La encuesta de percepciones acerca de la higiene de manos diseñada por la $\mathrm{OMS}^{10}$ se basa en una escala Likert de siete puntos entre "ineficaz" (1 punto) y "muy eficaz" (7 puntos), pero las respuestas tuvieron un comportamiento muy categórico hacia los valores más altos. Lo anterior hace muy inexacta una comparación de medianas y, por lo tanto, se compararon las proporciones de respuestas "muy eficaz" antes y después de la intervención. De nuevo, la no identificación de los formularios y de los mismos individuos impide una comparación pareada de McNemar y, por tanto, se realizó la prueba exacta de Fisher.

El indicador de cumplimiento con la higiene de manos se calculó como la proporción de oportunidades definidas por los cinco momentos (antes del contacto con el paciente, antes de tareas asépticas, después de riesgo de exposición a líquidos corporales, después del contacto con el paciente y después del contacto con su entorno), en las que realizaron acciones de lavado con agua y jabón o fricción de manos con un desinfectante alcohólico. La comparación de estas proporciones del "antes" y el "después" se hizo por medio de la prueba exacta de Fisher ante la imposibilidad de corrección por la no independencia de las observaciones, dado que un mismo trabajador podía ser observado en más de una oportunidad. 
En el hospital se realiza vigilancia activa de las IAAS y se utilizan las definiciones del CDC (Centers for Disease Control and Prevention) de Atlanta, Georgia, E.U.A.; con base en estos datos se calcularon las tasas por 1.000 días/ estancia. Las tasas de consumo de desinfectante alcohólico para las manos por 1.000 días/estancia se calcularon con base en la cantidad de producto despachado hacia los servicios desde el área de suministros. Los indicadores de consumo y de IAAS de antes y después de la estrategia se compararon por medio de un modelo regresión de Poisson, y para todas las comparaciones se consideró un valor de $\mathrm{p}<0,05$ como estadísticamente significativo.

\section{Resultados}

Durante la implementación se realizaron tres períodos de observación en cada una de las unidades. En el primer período durante los meses de junio y julio de 2009 se hicieron observaciones basales ("antes" de la intervención). El segundo y tercer períodos de observación se realizaron con posterioridad al entrenamiento ("después"), durante los meses de agosto-septiembre de 2009 y febrero de 2010 , respectivamente. En total, se hicieron 3.241 observaciones (1.279 antes del entrenamiento y 1.962 después) y se capacitaron en el modelo "Mis cinco momentos para la higiene de manos" a 999 trabajadores de la salud. El cumplimiento general con la higiene de manos antes de la intervención fue $82 \%$ y aumentó de manera significativa en el período posterior a $89 \%,(p=0,007)$.

Al hacer el análisis por categoría profesional y por unidades, esta tendencia se mantuvo en las auxiliares de enfermería, en las enfermeras profesionales de 83 a $90 \%$ $(p=0,001)$ y de 83 a $90 \%(p=0,018)$, respectivamente, en la unidad de aislamiento de 66 a $86 \%(\mathrm{p}=0,000)$ y en la unidad de cuidado intensivo de adultos de 82 a $88 \%$ $\mathrm{p}=0,032$ ).

En cuanto al cumplimiento durante cada uno de los cinco momentos, se observó un aumento significativo en las indicaciones "Antes del contacto con el paciente", "Antes de realizar una tarea aséptica", "Después del riesgo de exposición a líquidos" y "después del contacto con el entorno" (Tabla 1).

La encuesta de conocimientos se realizó a 299 trabajadores de las áreas seleccionadas, 216 (72\%) antes y 83 (28\%) después de la intervención educativa. El puntaje de conocimientos aumentó de manera significativa entre los dos períodos $(\mathrm{Me}=26, \mathrm{RIC}=22-28$ vs. $\mathrm{Me}=30$, $\mathrm{RIC}=26-32 ; \mathrm{p}=0,001)$. La encuesta de percepciones acerca de la higiene de manos se realizó a 246 trabajadores (73\%) antes y 92 trabajadores (27\%) después de la intervención. La mayoría de componentes de la estrategia fueron considerados "muy eficaces" antes de la intervención en más de $90 \%$ de las respuestas, con excepción de la retroalimentación ("los trabajadores reciben regularmente los resultados de su desempeño en la higiene de manos" $=78 \%$ ) y uno de los componentes de la cultura de seguridad del paciente ("se invita a los pacientes a que recuerden a los trabajadores la práctica de la higiene de manos" $=74 \%$ ). Después de la intervención, la percepción positiva se mantuvo sobre $90 \%$ en casi todos los ítems y hubo un aumento no significativo en la

Tabla 1. Cumplimiento con la higiene de manos antes y después de la estrategia multimodal (Hospital Pablo Tobón Uribe, Colombia, 2008-2010)

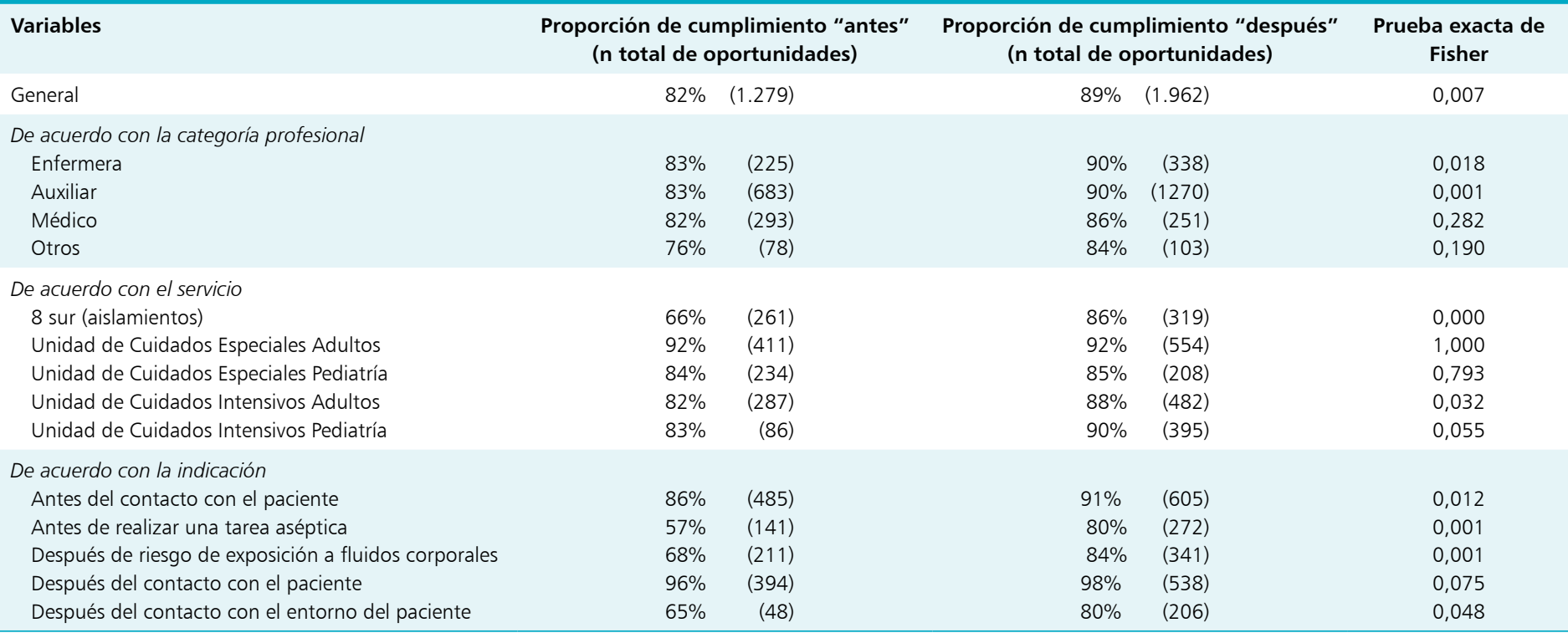


Tabla 2. Percepciones de los trabajadores de salud acerca de los cinco componentes de la estrategia de multimodal de la OMS (Hospital Pablo Tobón Uribe, Colombia, 2008-2010)

\begin{tabular}{|c|c|c|c|}
\hline Percepción & $\begin{array}{l}\text { Proporción de } \\
\text { respuestas "muy eficaz" } \\
\text { (antes, } n=246 \text { ) }\end{array}$ & $\begin{array}{l}\text { Proporción de } \\
\text { respuestas "muy eficaz" } \\
\text { (después, } n=92 \text { ) }\end{array}$ & $\begin{array}{c}\text { Prueba exacta } \\
\text { de Fisher }\end{array}$ \\
\hline \multicolumn{4}{|l|}{ Cambio en el sistema } \\
\hline En cada punto de atención se dispone de desinfectante alcohólico & $98 \%$ & $100 \%$ & 1,00 \\
\hline \multicolumn{4}{|l|}{ Educación } \\
\hline Cada trabajador tiene capacitación sobre la higiene de las manos & $96 \%$ & $99 \%$ & 0,053 \\
\hline Hay instrucciones para todos los trabajadores claras, simples y visibles sobre la higiene de manos & $95 \%$ & $98 \%$ & 0,748 \\
\hline \multicolumn{4}{|l|}{ Retroalimentación } \\
\hline Los trabajadores reciben regularmente los resultados de su desempeño en la higiene de manos & $78 \%$ & $89 \%$ & 0,028 \\
\hline Recordatorios en el sitio de trabajo & & & \\
\hline Se ponen carteles sobre la higiene de manos a modo de recordatorio en los puntos de atención & $96 \%$ & $100 \%$ & 0,142 \\
\hline \multicolumn{4}{|l|}{ Cultura de seguridad del paciente } \\
\hline Los directivos apoyan y promueven abiertamente la higiene de las manos & $93 \%$ & $97 \%$ & 0,455 \\
\hline Usted realiza perfectamente la higiene de las manos (siendo un buen ejemplo para sus colegas) & $93 \%$ & $96 \%$ & 0,495 \\
\hline Se invita a los pacientes a que recuerden a los trabajadores la práctica de la higiene de manos & $74 \%$ & $75 \%$ & 0,835 \\
\hline
\end{tabular}

percepción positiva sobre capacitación ("cada trabajador tiene capacitación sobre la higiene de las manos"), la cual aumentó a 99\% ( $\mathrm{p}=0,053)$, y en la retroalimentación ("los trabajadores reciben regularmente los resultados de su desempeño en la higiene de manos), la cual aumentó de manera estadísticamente significativa a $89 \%(p=0,028)$ (Tabla 2).

Las tasas de consumo de alcohol para higiene de manos incrementaron de manera constante y significativa en los cinco servicios en los que se implementó la estrategia, de 10,5 litros por 1.000 pacientes/día en el primer mes a 58,1 litros por 1.000 pacientes/día en el último mes [razón de incidencias $(\mathrm{RI})=2,39 ; 95 \% \mathrm{CI}=1,99 ; 2,88$ ], (Figura 1). Las tasas mensuales de IAAS, por otra parte, mostraron variaciones leves y no significativas en los mismos períodos de observación, (Figuras 2-4).

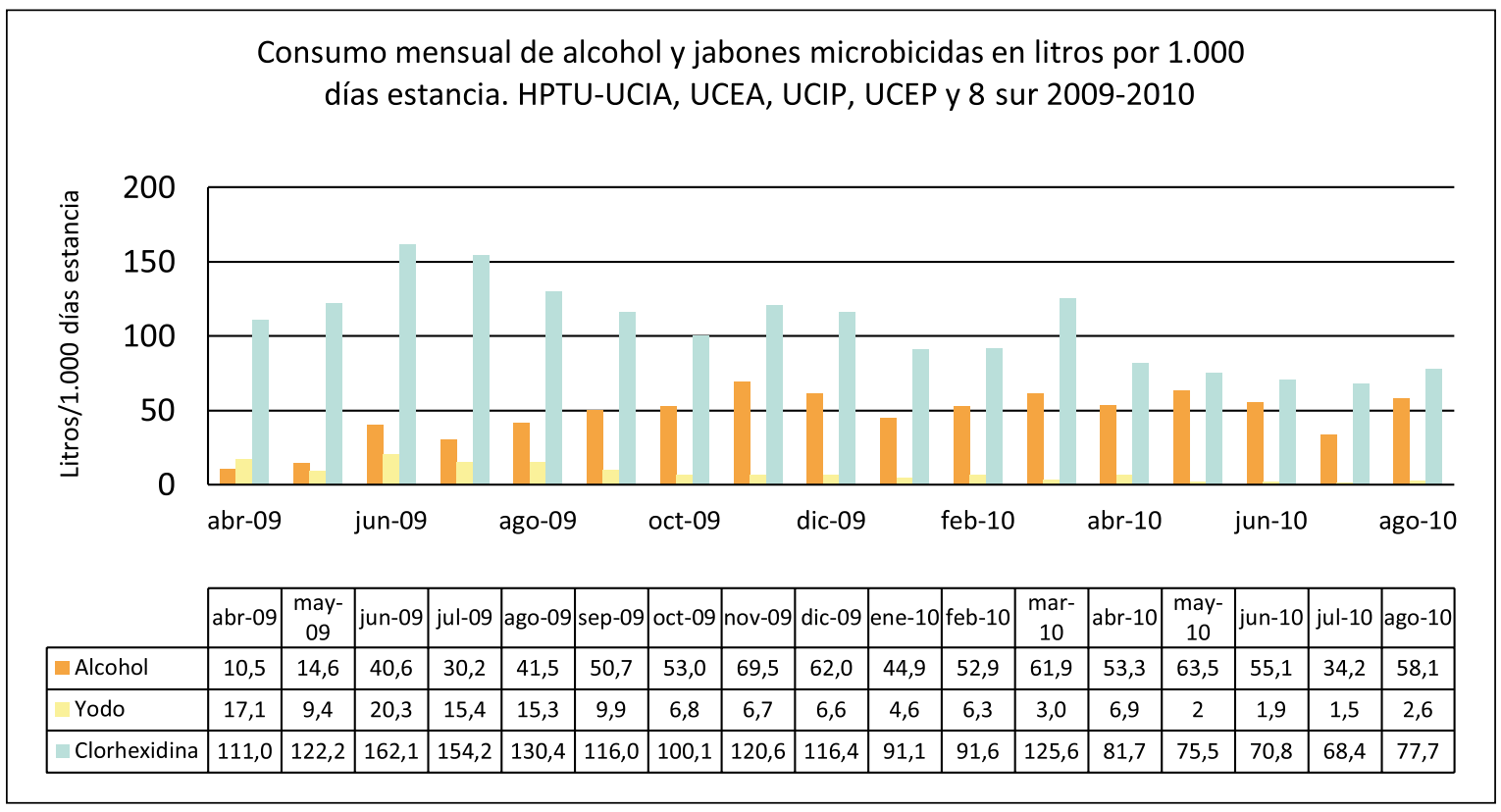

Figura 1. Tasa de consumo de alcohol y jabones microbicidas-Hospital Pablo Tobón Uribe, Colombia, 2009-2010. UCIA: Unidad de cuidados intensivos de adultos. UCEA: Unidad de cuidados especiales adultos. UCIP: Unidad de cuidados intensivos pediátricos. UCEP: Unidad de cuidados especiales pediátricos. 8 sur: Servicio de hospitalización para pacientes colonizados o infectados con microorganismos multi-resistentes. 


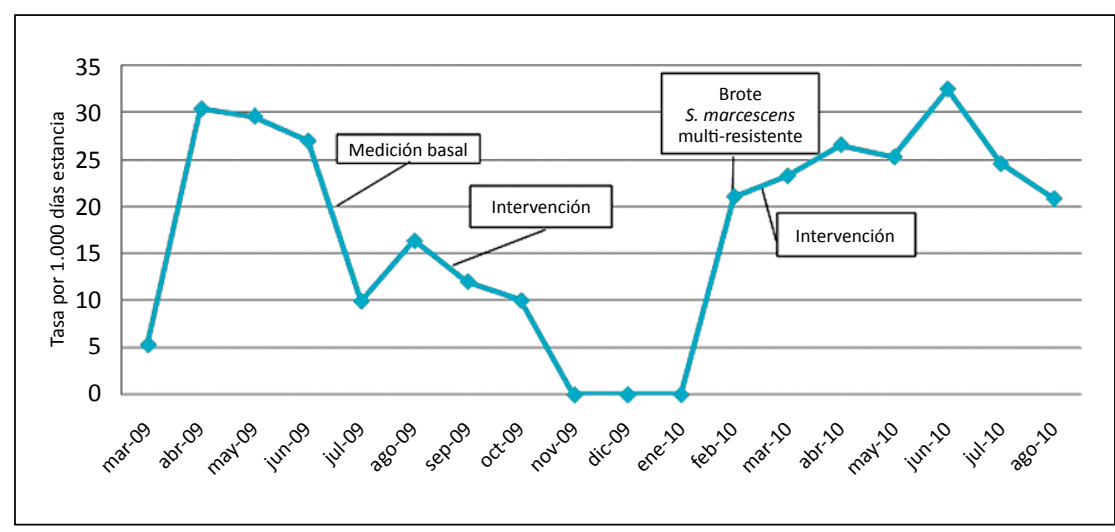

Figura 2. Tasa de infecciones asociadas a la atención en salud por mil días estancia-UCIP. Hospital Pablo Tobón Uribe, Colombia, 2009-20.

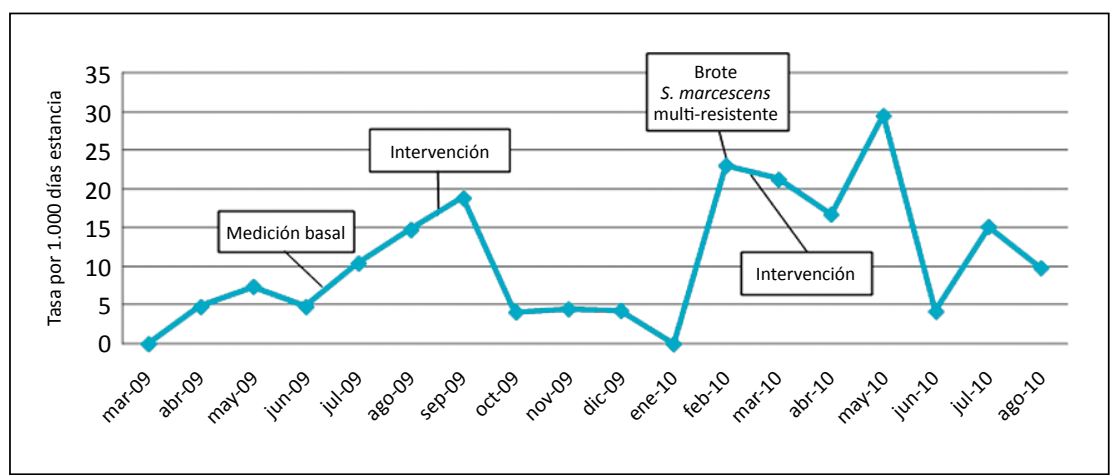

Figura 3. Tasa de infecciones asociadas a la atención en salud por mil días estancia - UCEP. Hospital Pablo Tobón Uribe, Colombia, 2009-2010.

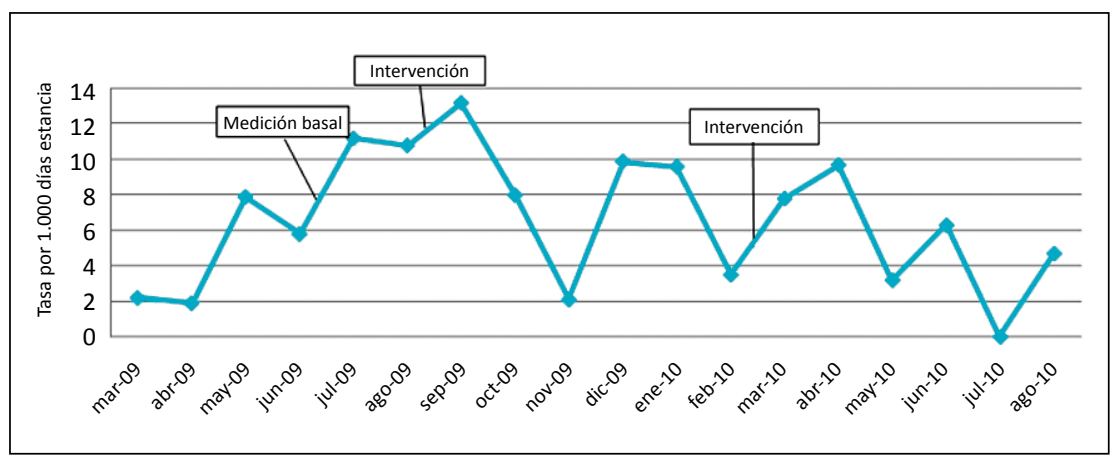

Figura 4. Tasa de infecciones asociadas a la atención en salud por mil días estancia - 8 sur. Hospital Pablo Tobón Uribe, Colombia, 2009-2010.

\section{Discusión}

La implementación de la estrategia "Atención Limpia es Atención Segura" en nuestra institución resultó en un aumento significativo del cumplimiento con la higiene de manos. Esta estrategia es amigable, estructurada y permite una medición organizada de los indicadores y la comparación de los datos a nivel mundial, ya que muchos hospitales en el mundo utilizan estas herramientas ${ }^{11-13}$. El cumplimiento basal fue alto ( $82 \%$ ), debido a que en la institución había en ese momento un brote de Klebsiella pneumoniae productora de carbapenemasas (KPC) y las unidades estudiadas estaban en aislamiento de contacto permanente. Además, los trabajadores estaban sensibilizados con las medidas para el control del brote, de las cuales la higiene de manos es una de las más importantes. Este fue el primer brote por KPC descrito en Colombia y tuvo un impacto importante en la dinámica del hospital. Inicialmente se identificó la infección por KPC en pacientes internados en las unidades de cuidados intensivos y para su control se implementó la creación de una unidad de aislamiento y seguimiento (cohorte). Esta fue la razón para elegir las unidades seleccionadas para empezar a desarrollar la estrategia. Todas las medidas implementadas, en conjunto con la estrategia multimodal, ayudaron al control del brote, con una disminución significativa en la tasa de infección y colonización por KPC, pasando de 1,27 en el segundo trimestre de 2008 a 0,08 en el segundo trimestre de $2011(\mathrm{p}<0,001)^{14}$.

Otros estudios demuestran que con la implementación de estrategias multimodales se puede aumentar significativamente el cumplimiento con la higiene de manos, independientemente del estado del desarrollo del país. En el Hospital du Point G de Bamako, Malí, con la estrategia de la OMS consiguieron aumentar el cumplimiento de $8 \%$ antes a 21,8\% después de la intervención $(p>0,001)^{11}$. En el Hospital de Niños de Westmead (Australia) lograron un aumento significativo desde $23 \%$ antes hasta $87 \%$ después de cinco años de intervención, y además reportaron un descenso significativo en las IAAS ${ }^{15}$. En el Hospital Johns Hopkins en Baltimore (E.U.A.), con la aplicación de un programa multimodal diseñado por ellos mismos, se observó un aumento del cumplimiento de 34 a $72 \%$, manteniendo estos niveles durante 20 meses de seguimiento ${ }^{16}$. En nuestro estudio no se logró documentar una reducción sostenida en las tasas de IAAS asociada al mejoramiento en el cumplimiento con la higiene de manos, debido al tiempo relativamente corto de seguimiento y a la magnitud en el aumento de dicho cumplimiento (diferencia absoluta de 7\%). Más aún, todas las observaciones que se realizaron durante el proyecto fueron advertidas y con retroalimentación inmediata, por lo que el cumplimiento puede verse "falsamente" aumentado por el efecto Hawthorne ${ }^{11}$, el que pudo influir en el alto porcentaje observado (89\%). A favor de esta hipótesis está el hallazgo de un porcentaje de cumplimiento de apenas $58,6 \%$ en observaciones inadvertidas que fueron realizadas de forma no sistemática por observadores entrenados y enmascarados durante el mes de septiembre de 2010. Aunque las observaciones advertidas pueden afectar el resultado obtenido, tienen la ventaja de facilitar 
la retroalimentación inmediata y, por lo tanto, el objetivo final de educar al trabajador de la salud, no sólo sobre la indicación en cada uno de los cinco momentos sino también en la técnica adecuada de fricción y lavado. El estímulo positivo es fundamental para el éxito de este tipo de estrategias, ya que el cumplimiento tiene mucho que ver con el comportamiento humano. El concurso realizado en el paso 3 de la estrategia fue una herramienta didáctica que ayudó a estimular a los trabajadores y fomentó el hábito de hacer higiene de manos en los cinco momentos, lo cual se reflejó en el aumento sostenido del cumplimiento.

El aumento observado en el consumo del desinfectante alcohólico fue importante y sostenido a lo largo del período de observación y era uno de los objetivos del proyecto. Hasta el momento no existe un estándar para la medición de este consumo ni tampoco un nivel ideal establecido. No obstante, el aumento en el consumo es una medida indirecta que se correlaciona con el cumplimiento de la práctica de higiene de $\operatorname{manos}^{17,18}$, que fue lo que se observó con la intervención en nuestra institución. En términos generales, la percepción de los trabajadores acerca de la estrategia fue altamente positiva y los ítems con puntuaciones bajas como la inclusión de pacientes y la retroalimentación periódica de datos sobre el cumplimiento se están interviniendo actualmente. Así mismo, los datos obtenidos de las encuestas de conocimientos se utilizaron para realizar la retroalimentación a los trabajadores en el paso 4. En el hospital se continúa con la implementación de la estrategia en otros 11 servicios, con un aumento, tanto en el cumplimiento de las acciones de higiene de manos como en el consumo de desinfectante alcohólico. También se consiguió mejorar el cumplimiento en las observaciones inadvertidas, alcanzando a $74 \%$ en 2012 , lo que demuestra que se fortalece el hábito de realizar higiene de manos. En conclusión, con la implementación rigurosa de la estrategia "atención limpia es atención segura" logramos un aumento significativo del cumplimiento con la higiene de manos.

\section{Resumen}

Introducción: La higiene de manos es una medida costo-efectiva para prevenir las infecciones asociadas a la atención de salud (IAAS). Para mejorar el cumplimiento se recomienda implementar estrategias multimodales como "atención limpia es atención segura" de la OMS. Objetivo: Estimar el efecto de la estrategia multimodal en cinco unidades de un hospital de tercer nivel en Medellín, Colombia (2008-2010). Métodos: Estudio cuasi-experimental antes y después. Resultados: El cumplimiento general con la higiene de manos aumentó de forma significativa en el período posterior a la implementación ( 82 a 89\%, p =0,007). El puntaje de conocimientos aumentó entre los dos períodos de tiempo (Mediana $=26$, $\mathrm{RIC}=22-28$ vs Mediana $=30, \mathrm{RIC}=26-32 ; \mathrm{p}=0,001)$. El consumo de alcohol aumentó de 10,5 litros a 58,1 litros por 1.000 pacientes/día [razón de incidencias $(\mathrm{RI})=2,39$; $95 \% \mathrm{CI}=1,99 ; 2,88]$. Las tasas mensuales de IAAS no mostraron variaciones $[\mathrm{RI}=0,90 ; 95 \% \mathrm{CI}=0,71 ; 1,13]$. Discusión: Este estudio demuestra que la implementación de una estrategia multimodal para la higiene de manos aumenta significativamente el cumplimiento con esta medida, independientemente del tipo de trabajador y el servicio hospitalario. Conclusión: Con la estrategia multimodal se aumentó significativamente el cumplimiento con la higiene de manos.

\section{Referencias bibliográficas}

1.- Report on the burden of endemic health care-associated infection worldwide. clean care is safer care. World Health Organization 2011. http://whqlibdoc.who.int/ publications/2011/9789241501507_eng.pdf

2.- Pittet D, Boyce J M. Hand hygiene and patient care: pursuing the Semmelweis legacy. Lancet Infect Dis 2001; 1: 9-20.

3.- Allegranzi B, Pittet D. Role of hand hygiene in healthcare-associated infection prevention. $\mathrm{J}$ Hosp Infect 2009; 73: 305-15.

4.- Pittet D. Improving compliance with hand hygiene in hospitals. Infect Control Hosp Epidemiol 2000; 21 (6): 381-6.

5.- Measuring hand hygiene adherence: overcoming the challenges. The Joint Commission 2009: pp 204.

6.- Trick W E, Vernon M O, Welbel S F, DeMarais P, Hayden M K, Weinstein R A.
Multicenter intervention program to increase adherence to hand hygiene recommendations and glove use and to reduce the incidence of antimicrobial resistance. Infect Control Hosp Epidemiol 2007; 28 (1): 42-9.

7.- Pittet D, Allegranzi B, Storr J. The WHO clean care is safer care programme: Field-testing to enhance sustainability and spread of hand hygiene improvements. J Infect Public Health 2008; 1: 4-10

8.- Ministerio de Salud y protección Social. República de Colombia. http://www. minproteccionsocial.gov.co/Documentos $\% 20$ y\%20Publicaciones/Forms/DispForm. aspx?ID=845 (accedido el 5 de septiembre de 2012).

9.- Guía para la aplicación de la estrategia multimodal de mejoramiento de la higiene de las manos de la OMS. Versión de Pruebas 1 2006-2007. Organización Mundial de la Salud 2007: pp 57.
10.- World Health Organization: clean care is safer care. http://www.who.int/gpsc/en/ (accedido el 5 de septiembre de 2012).

11.- Allegranzi B, Sax H, Bengaly L, Richet H, Minta D, Chraiti M, et al. Successful implementation of the World Health Organization hand hygiene improvement strategy in a referral hospital in Mali, Africa. Infect Control Hosp Epidemiol 2010; 31 (2): 133-41.

12.- Di Diodato G. An alternative methodology for interpretation and reporting of hand hygiene compliance data. Am J Infect Control 2012; 40: 332-5.

13.- Martín-Madrazo C, Soto-Díaz $\mathrm{S}$, Cañada-Dorado, Salinero-Fort M A, Medina-Fernández M, Carrillo de Santa Pau E, et al. Cluster randomized trial to evaluate the effect of a multimodal hand hygiene improvement strategy in primary care. Infect Control Hosp Epidemiol 2012; 33 (7): 681-8. 
14.- Correa A L, Restrepo A, Mazo L M, Valderrama M, Zuleta J J. Control of an outbreak of carbapenem-resistant Klebsiella pneumoniae in a tertiary care medical center (Colombia). In: Best practices in infection prevention and control: An international perspective. Soule BM, Memish ZA, Malani PN (eds). Second edition. Oakbrook Terrace. Joint Commission International 2012, pp 83-4.
15.- Jamal A, O'Grady G, Harnett E, Dalton D, Andresen D. Republished: Improving hand hygiene in a pediatric hospital: a multimodal quality improvement approach. Postgrad Med J 2012; 88: 353-8.

16.- Aboumatar H, Ristaino P, Davis R O, Thompson C B, Maragakis L, Cosgrove S. Infection prevention promotion program based on the PRECEDE model: improving hand hygiene behaviors among healthcare personnel.
Infect Control Hosp Epidemiol 2012, 33 (2): 144-51.

17.- Boyce J M. Measuring healthcare worker hand hygiene activity: current practices and emerging technologies. Infect Control Hosp Epidemiol 2011; 32 (10): 1016-28.

18.- Gould D J, Drey N S, Creedon S. Routine hand hygiene audit by direct observation: has nemesis arrived? J Hosp Infect 2011; 77: 290-3. 